\title{
Research on the Insulation Monitoring Devices for DC Power System Based on the Technology of AC Injection
}

\author{
Bai-chao WANG ${ }^{1 . a}$, Qiang YIN ${ }^{1, b}$, Xu-dan $\mathrm{GUO}^{2}$, Song-tao $\mathrm{LI}^{2}$, Hao YANG ${ }^{2}$, \\ Gang-lin $\mathrm{CAl}^{2}$ \\ ${ }^{1}$ XJ Power CO.,LTD., State Grid Corporation of China, Xuchang 461000,Henan,China \\ ${ }^{2}$ Stat Grid Sichuan Economic Research Institute, Chengdou 610000, Sichuan,China \\ awangbaichaody@126.com, byin-1-qiang@163.com, brenxiaodandy@126.com
}

Keywords: insulation monitoring devices; AC injection; DC bus; detection technology; grounding fault.

Abstract. In view of the problem of AC injecting into DC bus in the substation, the harm of AC injection is described, the characteristics of AC injection are analyzed, the detection technology of AC injection is researched, and the insulation monitoring devices for DC power system is designed. The kind of this grounding fault is prevented. A prototype test that it is able to monitor the effective value of AC injection in real time, send out the alarm information of AC injection and improve the reliability of the DC system, which has the advantages of simple operation, accurate measurement and good stability.

\section{Introduction}

DC system has a very important position in the substation and power plant. DC system is power equipment for the signal equipment, the protection automatic devices, the emergency lighting, the emergency power and the operating power supply. DC system should give service to the users of the hydraulic power plant, the thermal power plant, the substation and the other users using DC power equipment.

The secondary system is composed of the DC system and the AC system in the substation. The two systems are not connected to each other in the condition of normal operation, in which the coexistence of the AC and DC circuits is common. Recently, in view of AC injecting into DC bus, DC system and DC circuit are endangered, auxiliary relay maloperation is induced, the DC fuse is triggered, the substation blackouts and more serious consequences is resulted[1-3][7]. In order to avoid AC injecting into DC bus, the protecting maloperation is an urgent problem need to be solved.

It is pointed out that the grounding fault of AC injecting into DC has been prevented and the function of record and alarm on AC injecting into DC is need to be increased in the original insulation monitoring devices for DC power system[4]. When the DC system has an 10V or above effective value, the devices should be able to send out the AC injection alarm information, display the effective value of the AC injection and can choose the fault slip road [5].

Therefore, this paper presents the detection technology of AC injection and designs the insulation monitoring devices for DC power system, which can display the effective value of the AC injection in real time and can send out the alarm information of AC injection. A prototype test that the validity and feasibility of the devices are verified.

\section{The detection principle of AC injection}

The equivalent circuit diagram of DC system is shown in Fig.1. Where, R1 and R2 are the resistors in equalization bridge, $\mathrm{R} 3$ is the resistor in inspection bridge, $\mathrm{S} 1$ is the switch in inspection bridge, $\mathrm{R}+$ and $\mathrm{R}$ - are the resistors of positive and negative electrode to ground, $\mathrm{C}+$ and $\mathrm{C}$ - are the capacitances of positive and negative electrode to ground, and the imaginary frame is the insulation monitoring devices for DC power system. 


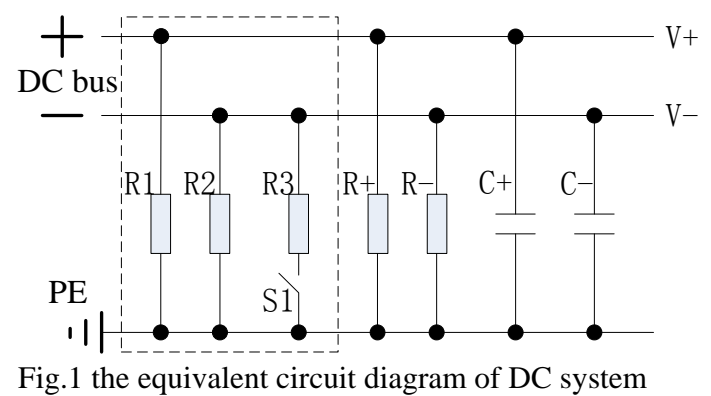

In the situation of AC injecting into DC system, the voltage of DC bus to ground will be a sinusoidal waveform, and the positive and negative voltage of the DC bus will not be changed. The equivalent circuit diagram of AC injecting into DC system is shown in Fig.2. Where, Vac is the effective voltage value of AC injection.

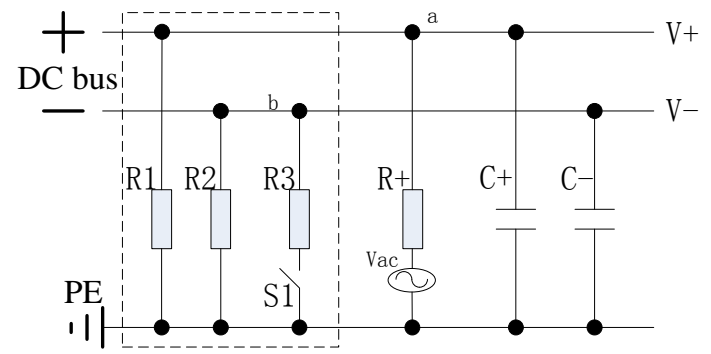

Fig. 2 the equivalent circuit diagram of AC injecting into DC system

For DC path, the DC system is shown to be the grounding fault of the positive electrode to the $\mathrm{R}+$, and the voltage of the positive electrode to ground is shown in formula (1).

$$
U_{a n}=U_{d c} *(R 1 / / R+/ / C+) /[(R 1 / / R+/ / C+)+(R 2 / / C-)]
$$

For AC path, the voltage of the positive electrode to ground is shown in formula (2).

$$
U_{a n}=U_{a c} * R+/[(R 1 / / C+) / /(R 2 / / C-)+(R+)]
$$

When the value of Udc and Uac is the fixed value, with the resistance of the $\mathrm{R}+$ is smaller, the grounding fault is more obvious for DC path, and the AC voltage value is higher for AC path. If $\mathrm{R}+$ is small, the DC voltage Ubn of the negative electrode to ground is close to the Udc, and the AC voltage Ubn of the negative electrode to ground is close to Uac. In this time, the peak voltage of the negative electrode to ground is up to Udc $+\sqrt{2}$ Uac. In the situation of AC injecting into DC system, Ubn and Uan values are monitored in real time, at the same time, the effective value of AC injection is the effective value of the cycle (Ubn-Udc) because of Ubn.

\section{The design of AC injection}

Hardware design is mainly divided into two parts. The first part is composed of the main control unit and the external device of the main control unit, the other part is composed of the sampling unit, the conditioning unit, and the alarm controll unit based on the DC bus and branch. The hardware block diagram is shown in Fig. 3.

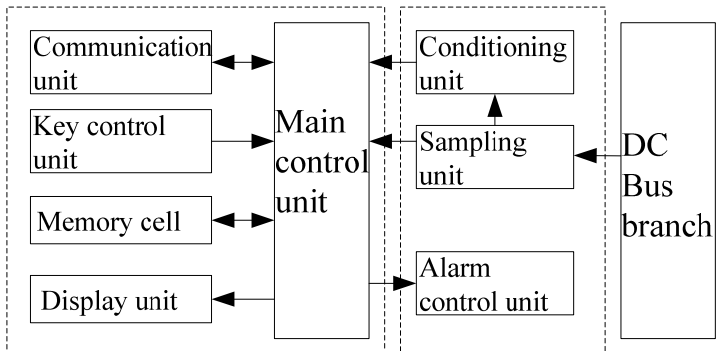

Fig. 3 the block diagram of the hardware

The hardware detection circuit of AC injection is shown in Fig.4. Where, Network labeled Samx can represent the sampling points $\mathrm{V}+$, the sampling points $\mathrm{V}$ - and the sampling points ground. They 
form the Sam_ADx signals into AD pin of the controller through the conditioning circuit, and then, the sampling value of the real-time detection is realized through the software operation.

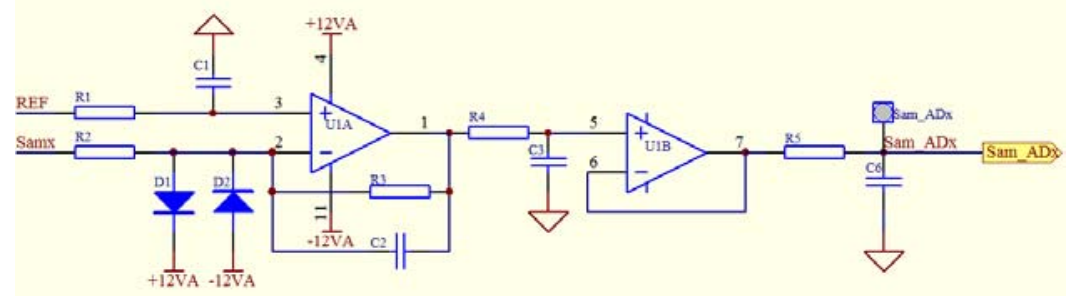

Fig.4 the hardware detection circuit of AC injection

\section{Experiment}

In order to verify the validity and reliability of the detection technology, the insulation monitoring devices for DC power system is designed. Test instruments has the FLUKE15B of the multimeter, the IRT7C of the multi function relay protection testing device and the MX30-3PI-400-LF-SNK of the programmable power supply. The positive electrode of AC injection voltage is shown in Table 1, and the negative electrode of AC injection voltage is shown in Table 2.

Table1: The positive electrode of AC injection voltage

\begin{tabular}{|l|l|l|l|l|l|}
\hline $\begin{array}{l}\text { Imposed } \\
\text { value(Vac) }\end{array}$ & 10 & 50 & 100 & 220 & 242 \\
\hline $\begin{array}{l}\text { Measured } \\
\text { value(Vac) }\end{array}$ & 10.0 & 49.9 & 99.9 & 220.2 & 243.2 \\
\hline error(\%) & 0 & 0.2 & 0.1 & 0.09 & 0.5 \\
\hline
\end{tabular}

\begin{tabular}{|l|l|l|l|l|l|}
\hline \multicolumn{7}{|c|}{ Table2: The negative electrode of AC injection voltage } \\
\begin{tabular}{|l} 
Imposed \\
value(Vac)
\end{tabular} & 10 & 50 & 100 & 220 & 242 \\
\hline $\begin{array}{l}\text { Measured } \\
\text { value(Vac) }\end{array}$ & 9.9 & 49.6 & 99.2 & 215.1 & 239.8 \\
\hline error(\%) & 1.00 & 0.8 & 0.8 & 2.23 & 0.91 \\
\hline
\end{tabular}

From table 1 to table 2, it can be seen that the error between the imposed value and the measured value is less than $3 \%$ in the range of $10 \mathrm{VAC} 242 \mathrm{VAC}$, and can accurately measure the effective value of AC injection. At the same time, when the effective value of $A C$ injection is greater than $10 \mathrm{VAC}$, the main control unit of the single chip microcomputer sends out the alarm information of $\mathrm{AC}$ injection, the indicator light of AC injection is open, and the device displays the effective value of AC injection.

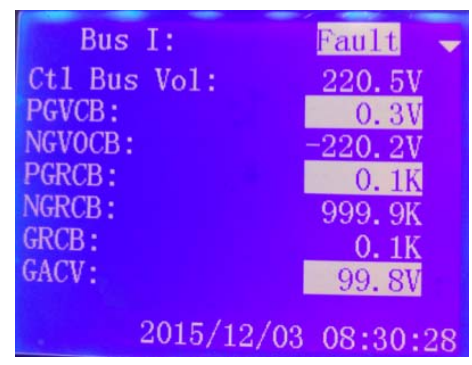

(a)

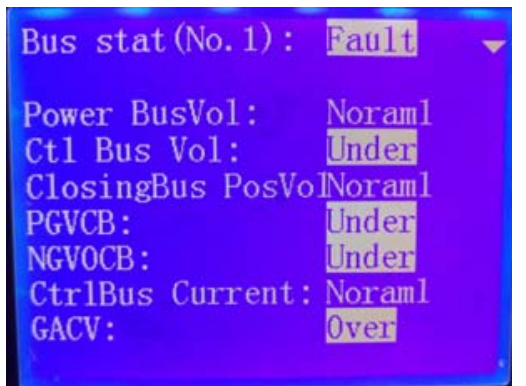

(b)

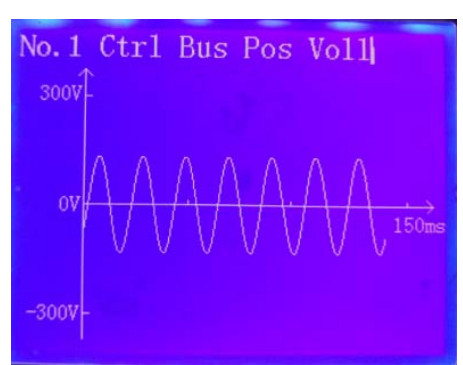

(c) 
The diagram of AC injecting into the positive electrode is shown in Fig.5, and then, it can be seen that the display status is given in the device displaying unit, and the conclusion is verified above the paper. The page of displaying the effective value of AC injection is shown in Fig.5(a). The page of the alarm information of AC injection is shown in Fig.5(b). The page of the voltage curve of AC injection is shown in Fig.5(c). The case of AC injecting into the negative electrode is same to AC injecting into the positive electrode.

\section{Conclusions}

In this paper, a detection method of AC injection is presented. The insulation monitoring devices for DC power system is designed. A prototype test that it is able to monitor the effective value of AC injection in real time, send out the alarm information of AC injection. This device has been running for more than half a year in the field of substation, with the advantages of reliable, accurate, stable and innovative, at the same time, with strong market competitiveness.

\section{Acknowledgements}

This work was financially supported by Science and Technology Project of State Grid Corporation of China(Research on the intelligent on-line monitoring and maintaining technology for AC-DC integration power in substation).

\section{References}

[1] TANG Jian-hong, SU Wen-bo, PAN Xiang-hua. Research of non-fault trip accident in 500kV substation[J]. Electric Power Automation Equipment, 2005.25(12):77-79

[2] MENG Fan-chao, GAO Zhi-qiang, YANG Shu-dong. Analysis of the tripping of the circuit breaker by the AC in the DC[J]. RELAY, 2007.35(14):77-78

[3] The 18 items of grid major anti accident measures of SGCC[Z]. Beijing: China Electric Power Press. 2011Y.

[4] DL/T 1392-2014 Technical specification of insulation monitoring devices for DC Power system[S].

[5] HUANG Dong-shan, ZHOU Wei, YANG Li-cai, etal. Study on insulation failure and loop fault in the DC system[J].Electronic Test, 2014.24:130-132

[6] DING Xiao-bing, ZHAO Man-yong, PI Xian-song, etal. Treatments to avoid misoperation of busbar protection when AC flows into DC circuit[J]. Power System Protection and Control, 2008.36(2):97-99

[7] WEI Bin. Failure test analysis of AC fleeing into the DC system[J]. Electric Power Science and Engineering, 2013.29(3):29-32 